\title{
Herpes Zoster and Subsequent Cancer Risk
}

\author{
Shih-Wei Lai ${ }^{a, b}$ Yu-Hung Kuo ${ }^{c}$ Kuan-Fu Liao ${ }^{d, e}$ \\ ${ }^{a}$ College of Medicine, China Medical University, Taichung, Taiwan; ${ }^{b}$ Department of Family Medicine, China Medical \\ University Hospital, Taichung, Taiwan; ' Department of Research, Taichung Tzu Chi Hospital, Taichung, Taiwan; \\ ${ }^{\mathrm{d}}$ College of Medicine, Tzu Chi University, Hualien, Taiwan; ${ }^{e}$ Division of Hepatogastroenterology, Department of \\ Internal Medicine, Taichung Tzu Chi Hospital, Taichung, Taiwan
}

Dear Editor,

Epidemiological studies have shown that patients with cancer are at risk of developing herpes zoster $[1,2]$, but no consistent association has been shown between herpes zoster and subsequent cancer risk. Recently, a nationwide retrospective cohort study in the Republic of Korea conducted by Kim et al. [3] published in Dermatology reported that the overall incidence rate of subsequent cancer was similar between patients with and without herpes zoster (7.62 vs. 7.66 per 1,000 person-years), which was compatible with previous research [4]. The study by Kim et al. [3] added marked value to the current literature and indicates future research direction.

In order to test this issue in a different population, a preliminary nationwide cohort study was conducted using the 2003-2012 database of the Taiwan National Health Insurance Program with 23 million residents living in Taiwan $[5,6]$. There were 17,624 subjects aged 20 84 years with newly diagnosed herpes zoster in the herpes zoster group and 35,248 randomly selected sex-matched and age-matched subjects without herpes zoster in the non-herpes zoster group. Subjects with a history of any cancers before the index date were excluded from the study. Table 1 demonstrates that the overall incidence rate of subsequent cancer was 1.16-fold higher in the herpes zoster group than in the non-herpes zoster group (1.07 vs. 0.92 per 100 person-years, $95 \%$ confidence interval [CI] 1.07-1.27; $p=0.001$ ). As stratified by sex and age, the incidence rates of subsequent cancer were all higher in the herpes zoster group than in the non-herpes zoster group. The incidence rates of subsequent cancer increased with age in both groups, the highest being in the herpes zoster group aged 65-84 years (1.98 per 100 person-years). Although the incidence rate of subsequent cancer was higher within 1 year of herpes zoster diagnosis when compared with the non-herpes zoster group (14.09 vs. 12.47 per 100 person-years), it did not reach statistical significance (95\% CI $0.92-1.39 ; p=0.24$ ). The incidence rate of subsequent cancer appeared to be statistically higher after 1 year of herpes zoster diagnosis, compared with the non-herpes zoster group ( 0.89 vs. 0.77 per 100 person-years, 95\% CI 1.06-1.27; $p=0.002$ ).

Our study demonstrates that the relative risk for subsequent cancer was modestly higher in the herpes zoster group (incidence rate ratio 1.16), which is compatible with a meta-analysis showing that the relative risk for subsequent cancer was 1.42 (95\% CI 1.18-1.71) among herpes zoster patients [7]. We noted that the risk of sub- 
Table 1. Incidence rate of subsequent cancer between adults with and without herpes zoster

\begin{tabular}{|c|c|c|c|c|c|c|c|c|c|c|}
\hline Variable & \multicolumn{4}{|c|}{ Herpes zoster } & \multicolumn{4}{|c|}{ Non-herpes zoster } & $\operatorname{IRR}(95 \% \mathrm{CI})^{\mathrm{a}}$ & $p$ value \\
\hline \multicolumn{11}{|l|}{ Sex } \\
\hline Men & 8,092 & 434 & 35,831 & 1.21 & 16,184 & 773 & 72,144 & 1.07 & $1.13(1.01-1.27)$ & 0.041 \\
\hline Women & 9,532 & 399 & 42,117 & 0.95 & 19,064 & 669 & 84,818 & 0.79 & $1.20(1.06-1.36)$ & 0.004 \\
\hline 40-64 years & 9,273 & 360 & 41,471 & 0.87 & 18,546 & 605 & 83,457 & 0.72 & $1.20(1.05-1.36)$ & 0.007 \\
\hline 65-84 years & 5,156 & 441 & 22,256 & 1.98 & 10,312 & 796 & 44,952 & 1.77 & $1.12(1.00-1.26)$ & 0.058 \\
\hline \multicolumn{11}{|l|}{ Follow-up period } \\
\hline$<12$ months & 2,244 & 146 & 1,036 & 14.09 & 4,451 & 248 & 1,989 & 12.47 & $1.13(0.92-1.39)$ & 0.240 \\
\hline$\geq 12$ months & 15,380 & 687 & 76,912 & 0.89 & 30,797 & 1,194 & 154,973 & 0.77 & $1.16(1.06-1.27)$ & 0.002 \\
\hline
\end{tabular}

Incidence rate: per 100 person-years. IRR, incidence rate ratio; CI, confidence interval. ${ }^{a}$ IRR: herpes zoster versus non-herpes zoster.

sequent cancer was statistically higher among those with a diagnosis of herpes zoster for 1 year or longer. Due to the good quality of the Taiwan medical system, it did not need to take 1 year to make a diagnosis of cancer since the onset of cancer-related symptoms. Therefore, spending 1 year or longer to make a diagnosis of cancer seemed to be a long time since the presentation of herpes zoster. It is not a reasonable assumption that herpes zoster is associated with an increased risk of developing cancer, but whether herpes zoster could be a marker of occult cancer needs confirmation from other real-world data. Currently, there is no definite evidence to support screening all cancers among those with a new diagnosis of herpes zoster.

\section{Key Message}

Currently there is no definite evidence to support screening all cancers among those with a new diagnosis of herpes zoster.

\section{Disclosure Statement}

The authors have no conflicts of interest to declare.

\section{Author Contributions}

Shih-Wei Lai contributed to the conception of the article, initiated the draft of the article, and approved the final draft submitted. Yu-Hung Kuo and Kuan-Fu Liao conducted the data analysis.

\section{References}

1 Liao KF, Kuo YH, Lai SW. Increased Risk of Herpes Zoster in Cancer Patients. J Infect Dis. 2019 Sep;220(9):1542-3.

2 Qian J, Heywood AE, Karki S, Banks E, Macartney K, Chantrill L, et al. Risk of Herpes Zoster Prior to and Following Cancer Diagnosis and Treatment: A Population-Based Prospective Cohort Study. J Infect Dis. 2019 Jun;220(1):3-11.
3 Kim M, Han K, Yoo SA, Lee JH. Herpes Zoster and Subsequent Cancer Risk: A Nationwide Population-Based Cohort Study in Korea. Dermatology. 2020. DOI: 10.1159/ 000505911.

4 Wang YP, Liu CJ, Hu YW, Chen TJ, Lin YT, Fung CP. Risk of cancer among patients with herpes zoster infection: a population-based study. CMAJ. 2012 Oct;184(15):E804-9.

5 Liao KF, Lin CL, Lai SW. Association between colorectal cancer and thiazolidinediones administration in a case-control study. Biomedicine (Taipei). 2019 Mar;9(1):4.
6 Ministry of Health and Welfare Taiwan. 2018 Taiwan Health and Welfare Report [cited 2020 May 1]. Available from: http://www. mohw.gov.tw. [English version].

7 Schmidt SA, Mor A, Schønheyder HC, Sørensen HT, Dekkers OM, Cronin-Fenton D. Herpes zoster as a marker of occult cancer: A systematic review and meta-analysis. J Infect. 2017 Mar;74(3):215-35. 